\title{
Exception Handling in Communication Protocols
}

\author{
M. Stella Atkins
}

Computer Science Department, The University of British Columbia.

This paper describes structures used for exception handling in multi-process implementations of the $\times .25$ and $X .29$ protocols. Each protocol layer is implemented as a collection of cooperating processes. These are structured in one of two alternative ways, reflecting differences in the type of exceptional situations that may occur.

Within each of the lower protocol layers, one process takes the form of a Finite State Machine. In these lower level protocols, where it is difficult to identify "normal" transitions, there are no special exception cases; we treat all transitions as being equally likely to occur.

For higher level protocols such as the $\times .29$ protocol, exceptions occur less frequently. Here. transmitting and receiving are performed by two independent processes, and exception handling is performed by an auxiliary process which is invoked as needed. This multi-process structure matches the functionality of the protocol, and results in software which is easy to program and easy to understand.

\section{Introduction}

The CCITT Recommendation X.25 [2] defines a multi-layer protocol for interfacing computer equipment to a packet-switched network. Each $\times .25$ protocol layer can be described as a Finite State Machine (FSM) making appropriate state transitions on receipt of packets or timeouts [1].

The CCITT's Virtual Terminal Protocol X.29 [6], here referred to as Datapac's Interactive Terminal Interface protocol (ITI), establishes a data structure that it views as representing the terminal. A remote terminal user may therefore interface with a host-side application program over the Datapac network via the host-side $X .25$ and ITI protocols. These protocols have been successfully implemented on a small timesharing system using a multi-process operating system called Verex, linking it to Canada's Datapac network.

Permission to copy without fee all or part of this material is granted provided that the copies are not made or distributed for direct commercial advantage, the ACM copyright notice and the title of the publication and its date appear, and notice is given that copying is by permission of the Association for Computing Machinery. To copy otherwise, or to republish, requires a fee and/or specific permission.
The Verex operating system [8] is a descendent of Thoth[3], in which processes communicate via messages. The message-passing process model is a simple and unifying approach to synchronisation, data transfer, concurrency, and distribution of function across processors and memories.

Using multiple processes communicating via messages such as are found in the Thoth and Verex operating systems it is possible to model the action of the lower-level protocol finite state machines. When no special exception cases are identifiable, the process executing the Finite State Machine can then be conveniently implemented as a combination of a "server" process and a Finite State Machine. This process provides all the necessary synchronisation of transmitting and receiving, leading to efficient handling of all transitions. Deering has shown [7] in his $\times .25$ implementation using Verex that such structuring is feasible. The resulting software is produced quickly and models closely the protocol descriptions contributing to its understandability and maintainability .

Scotton applied similar techniques [9] for his implementation of the ITI protocol in Verex, but recognized several disadvantages in using these techniques for the higher level protocols. We therefore propose a new structure for higher level protocols, where exceptional cases appear less frequently, and a "normal" case can be identified.

In this paper we first outline the process and message-passing environment supported by Verex. We then illustrate how this environment can be used to provide implementations of the $\times .25$ protocol, and describe Deering's implementation. We discuss the exception handling mechanisms used in this implementation, where exceptions are treated as the rule, and the problems in extending this to an implementation of a higher-level protocol such as the ITI protocol.

We then describe the novel exception-handling mechanism employed in the ITI implementation. This structure employs separate independent processes for transmitting and receiving together with an auxiliary exception-handling process to coordinate transmitting and receiving when an exception occurs.

We show how this structure overcomes the problems of the server model, and conclude with a summary of the major aspects of the design. 


\section{Implementation Environment}

This section is abstracted from [6], and is included for readers unfamiliar with the Thoth and Verex operating systems. Verex is structured as a kernel and a collection of processes [8]. The kernel provides process creation and destruction, interprocess message-passing and low-level device support. Processes provide the bulk of the operating system services, such as memory management, a file system, terminal support, interactive session management, and network access. Application programs execute as processes which obtain services by sending messages to system processes.

Central to the design of Verex is the use of multiple processes to support concurrent and asynchronous behaviour. To make multi-process structuring attractive, the implementation guarantees that processes are cheap to create and destroy, that process switching is efficient, and that message-passing is fast. Short, fixed-length messages are sent directly to processes - there are no connections and no ports. Each process has a unique, global identifier and a single FIFO queue for incoming messages.

The kernel provides the following message-passing primitives:

\section{Send ( message, id )}

sends the specified message to the process identified by id and suspends the sending process until a reply is received. The contents of message are changed by the reply.

$$
\text { id = Receive ( message ) }
$$

retrieves the first message from the incoming message queue and returns in id the process identification of the sender. If the queue is empty, the receiver is suspended until a message arrives.

\section{Reply ( message, id )}

returns a reply message to the process identified by id. This enables the sender to resume execution.

The Send, Receive and Reply message primitives, although not the only message primitives supported within Verex, form the basis for most of the interprocess communication.

The message-passing primitives described above support the remote procedure call style of interprocess communication. Sending a message and awaiting its reply is much like passing arguments to a procedure and receiving its results. However, processes that receive messages are more powerful than procedures: they can reply to the messages in any order, or even forward them to other processes for reply.

These message facilities are often used to establish a client/server relationship between processes, as described by Cheriton [5]. A client process requests some service by sending a message to a server process, and awaiting a reply. The request message contains all the necessary fields to specify the service required; the reply message contains the results of servicing the request. The client is given the simple procedure-like interface of Send, whereas the server can use the greater flexibility of a receiver to provide sophisticated scheduling and interleaving of services.

An example of a server-based facility is the Verex I/O system [4]. Processes obtain input and generate output by sending requests to file servers. A file server is any process which implements the Verex file access interface; it may provide disk storage, device access, or any other source/sink of data that can fit the model. All system servers, including file servers are structured so that they execute a repeated loop; first waiting to Receive any message, then processing it quickly so as not to block receipt of other messages.

\section{Implementation of Finite State Machines}

There are at least two alternative techniques for implementing a FSM using multiple processes - as a so-called sequential automaton, or as a Verex-like server. These are well known to compiler writers, and the implications of their use in implementing protocol FSM's are described briefly below.

\subsection{Finite State Machine as a sequential automaton}

In this method of implementing a FSM, a "normal" set of states and transitions is identified, such as states $1,2,3$ and 4 . The FSM is implemented by coding the transitions from one normal state to the next normal state as sequential statements. All other transitions and states are treated as exceptional, in the sense that they are non-normal. On encountering a message which would cause a non-normal transition, the FSM undergoes a transition to an exceptional state. In the implementation, control is diverted to the section of code handling this exception, and eventually control is returned to the appropriate place in the normal flow.

The advantages of this kind of implementation are the clear distinction between "normal" and "non-normal" transitions, and the readability of the control flow in the sequence of statements.

However, the technique has two major disadvantages. First, it involves the use of "exception trapping" mechanisms, including long jumps to transfer flow of control to other non-sequential states, with appropriate context-saving and restoring. Second, as Deering points out in [7],

"In implementations of a full duplex protocol for an error-prone circuit, it is difficult and perhaps meaningless to identify the "normal" flow of control exceptions are the rule."

\subsection{Finite State Machine as a server}

An alternative to the above technique for implementing a FSM is to encode the current state explicitly in a global state vector instead of in the current position in a sequence of statements (the - program counter). The control flow is implicit in the sequence of values taken on by the state variables. The 
FSM may then be coded as a server executing a repeated loop consisting of a single Receive statement followed by a conditional branch depending on the message type and state in combination. This conditional branch may contain as many permutations of state and message type as required.

This FSM structure sacrifices some readability but avoids the use of exception-trapping mechanisms. It also avoids problems associated with identifying the "normal" flow of control - all states and messages are treated as equally likely.

Two major factors influenced our choice of this structure for our $\times .25$ implementation. First, the usual form for servers is that all the messages are received at a single place, and that subsequent processing of the message is determined by a branch on the message type. We had a great deal of experience with such servers, which would be helpful in using this design for our $\times .25$ implementation. Second, in the $\times .25$ protocol, certain messages such as the "Clear" request, cause the protocol FSM to return to the same state, regardless of what state it was in before. The FSM modelled as a server with an initial branch on message type would reflect this situation cleanly and efficiently.

\section{The $\times .25$ implementation}

The process structure of the Verex $\times .25$ packet-level protocol implementation is shown below in Fig.l.

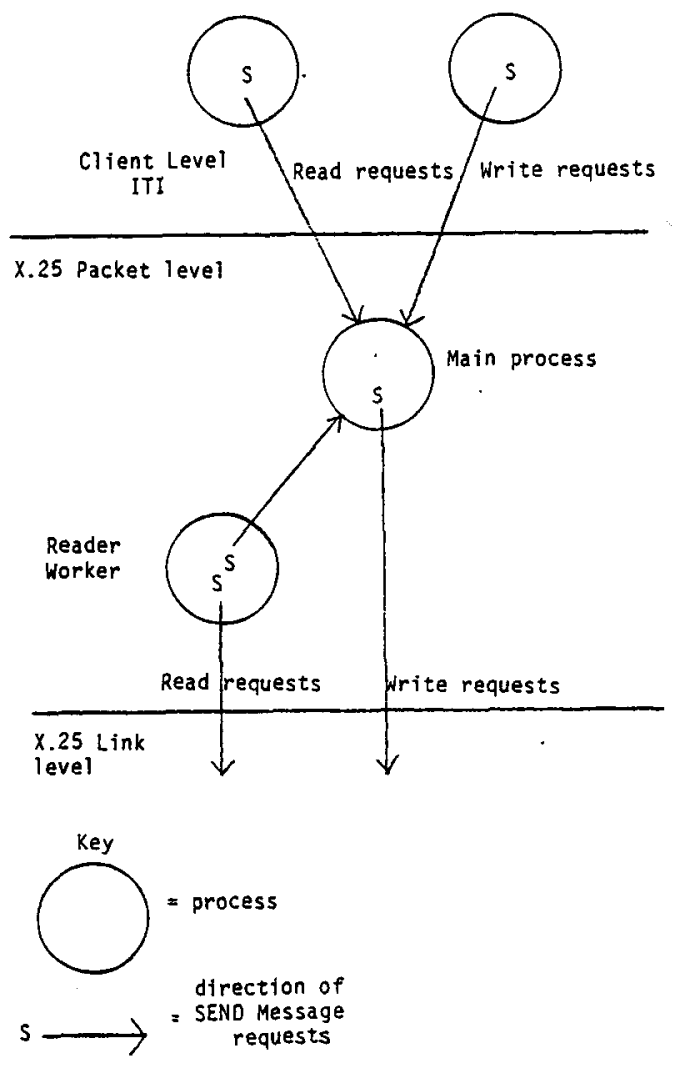

Figure 1. $X .25$ process structure
The Main process acts as a Verex server, accepting client requests for service and also accepting notifications from its reader worker when data is ready. The reader worker is used to await data from the protocol layer below; it prevents the Main process from being blocked on the data. The Main process also acts as the protocol FSM, changing state according to the data received from the reader worker. A writer worker process was not used in this implementation because the lower level protocol guaranteed to reply immediately to write requests, so the Main process would not block on a write for any length of time.

The Main $\times .25$ process is structured as shown in Fig.2.

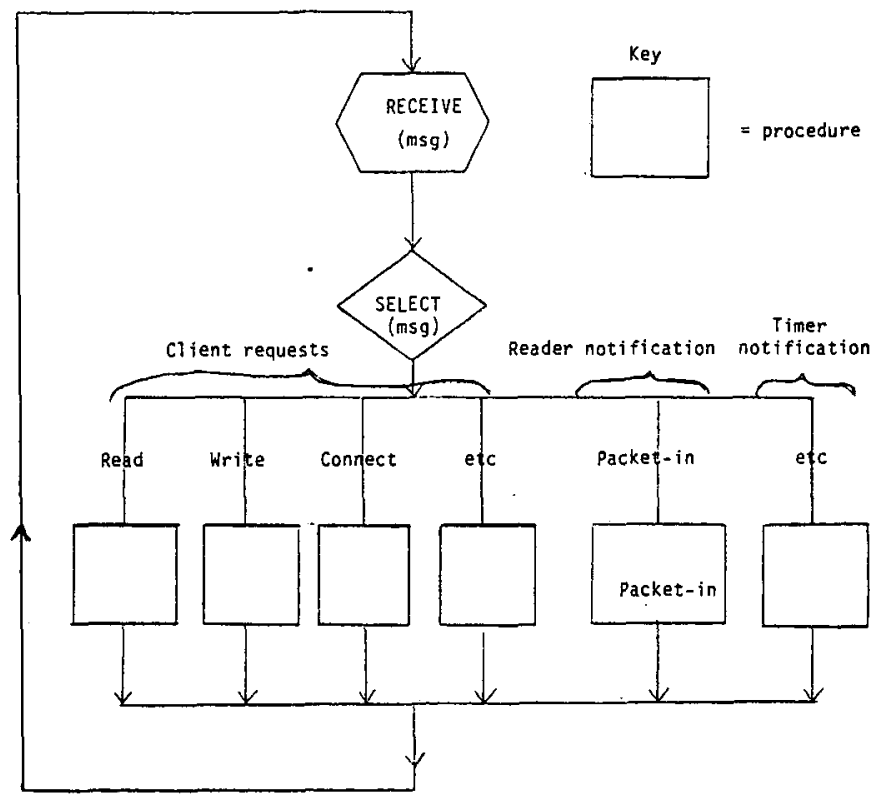

Figure 2. Structure of the $X .25$ server

Within the procedure Packet_in, the $X-25$ server is coded as a protocol FSM, branching on each combination of state and message type as shown below in Fig.3.

The exception situations are therefore treated as efficiently as the normal situations - no special context-saving and recovery is needed, nor are any longjumps performed.

\section{The ITI implementation as a server}

Having succesfully implemented the $X .25$ protocol as a FSM server, we tried to apply the same tecnnique to a higher-level protocol. The Datapac Interactive Terminal Interface (ITI) [DATA78] was initially implemented in this way by Scotton [SCOT81]. ITI acts mainly as an $I / O$ filter, transmitting read/write requests from its client (called the application) through to X.25. In this implementation exceptions occur when the underlying network fails in some way, or when the remote user wishes to communicate a "break" signal. As with $\times .25$, the ITI Main process executes a repeated loop with a single Receive statement. It maintains the 


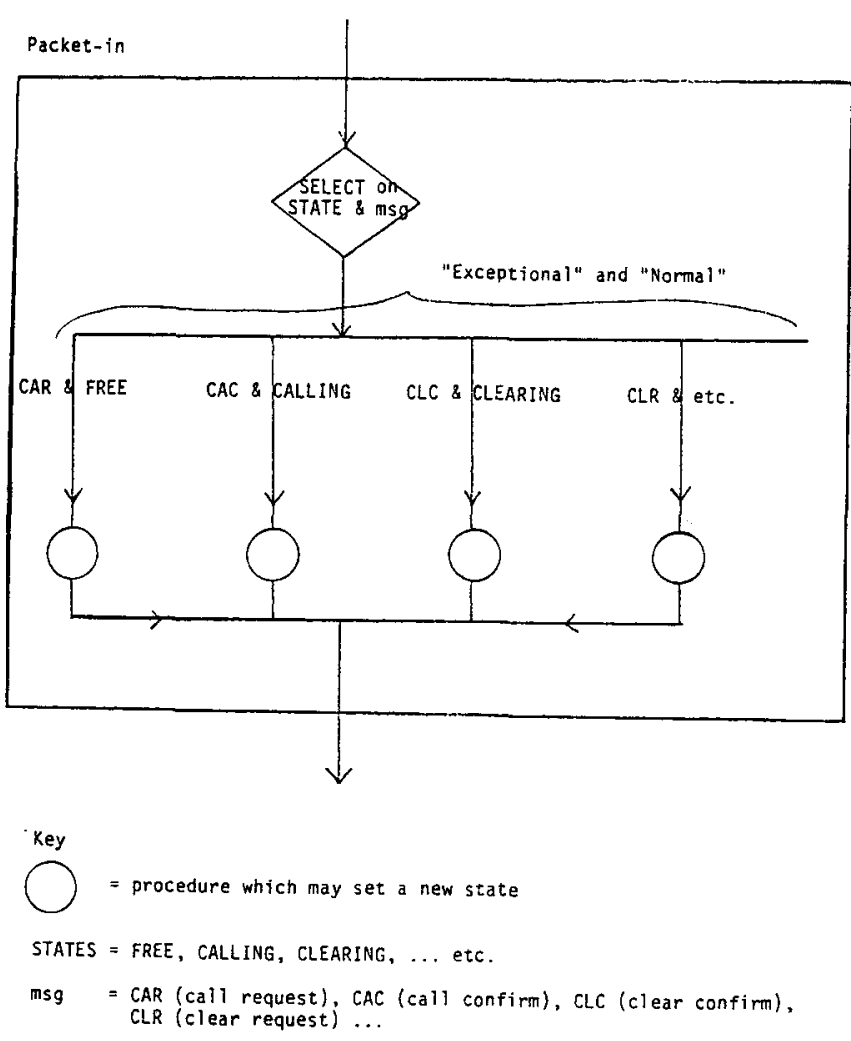

Figure 3. Structure of the X.25 FSM

state vector which is updated as events occur. Extra worker processes, a reader and a writer, are required to handle delays in reading and writing to $X .25$.

Although the network can fail at any time, it is not considered necessary for the ITI server to be informed of this event immeditely (i.e. asynchronously). Instead, the application is unaware of any disruption to the i/o service and executes normally until a read or write operation is requested. When this occurs, the ITI reader worker receives, synchronously, the exceptional information concerning the failure of the underlying network layer. The ITI server attempts to provide error recovery from this situation, by allowing the user to reconnect by logging in again. The application is blocked until either the transport connection is re-established, in which case the application continues normall, or until a timeout occurs, when an end-of-file condition is returned to the application.

This synchronous scheme cannot however be used to communicate a break signal from the remote user, as notification cannot wait until the user requests an i/o operation. For example, if a user initiates a long compilation, then notices an error and presses the break key, a response is required within a short finite period, say half a second.

\subsection{Process destruction for asynchronous exceptions}

There are several alternatives for handling this kind of exceptional, asynchronous event. The most obvious is for the $X .25$ server to send a message to the main ITI process. However, this kind of upward communication from server to client is not allowed in
Verex, because of potential deadlock or abuse. The usual practise in Verex is to use process destruction. In this technique, the lower level process is given the process id of a "victim" to be destroyed when an exception occurs. The higher level process receives notification of this destruction through another process, an "undertaker", which is blocked explicitly awaitin the victim's death.

A variant of this scheme was used in Scotton's implementation of ITI but it was considered to be clumsy, involving two extra processes whose only function was to relay a message from the lower level to the ITI Main process (which is usually blocked awaiting any message). Also the overhead of process destruction and recreation was not insignificant.

\subsection{Interrupt switch for asynchronous exceptions}

A second solution for handling asynchronous exceptions, suggested by Cheriton, was devised where ITI put itself in the position of being always ready to receive a message from the lower level, regardless of whether or not the application had a read request outstanding. This was acheived by adding another state variable to both the ITI and $X .25$ servers. This acted as a switch to exert flow control on the movement of data from the lower level $\times .25$ to the higher level ITI. When the switch was "ON", $\times .25$ would return both data and interrupts; when "OFF", $X .25$ would return break signals only. Unfortunately, this scheme incurs the overhead of 4 extra process switches on each read request - 2 to set the switch on and off. This overhead can be reduced by setting a default for $X .25$ to set the switch "OFF" automatically after satisfying every read request. Then ITI's Main process controls the switch setting so that when there is an application level read request, it requests data from $\times .25$ setting the switch "ON".

This solution has been successfully implemented; however, there are certain disadvantages with this scheme for asynchronous exception-handling. First, the solution is specific to the ITI-X.25 interface - it can't be generalised to solve other asynchronous communication problems. Second, we have two separate mechanisms for exception situations - those encountered synchronously and treated as first level branches by the FSM, and those encountered asynchronously. Third, there is an overhead of two extra process switches for each read request from ITI to $\times .25$.

\subsection{An exception-handler process for asynchronous exceptions}

We therefore designed a new solution for handling exceptional asynchronous events encountered by ITI.

This solution requires an extra "exception handler" process $E H$, which registers its process id with the lower level process. Registration is similar to the notification of the victim's id in the initial scheme. EH then blocks awaiting an exceptional event to occur at the lower level by performing a Send request to the lower level process.

When an asynchronous exception occurs (such as an interrupt from the remote user), $\times .25$ makes a 
Reply to EH. EH then takes control as necessary, by initiating further read requests via the reader worker.

\section{The ITI implementation as an $1 / 0$ filter}

Having designed an exception handling process to handle asynchronous exceptions encountered by ITI, we observed that the other type of exceptional event that of failure of the underlying network - could also be efficiently handled using an exception handler process. Further, the same exception handler process could be used to handle both types of exception reported from the lower level.

We then found that the two major functions of ITI's Main process - that of coordinating action on receiving interrupts and that of reconnection - were taken over by the exception handler process.

We therefore decided to restructure the ITI processes as a reader worker, a writer worker, a timer and an exception handler process, as shown below in Fig. 4.

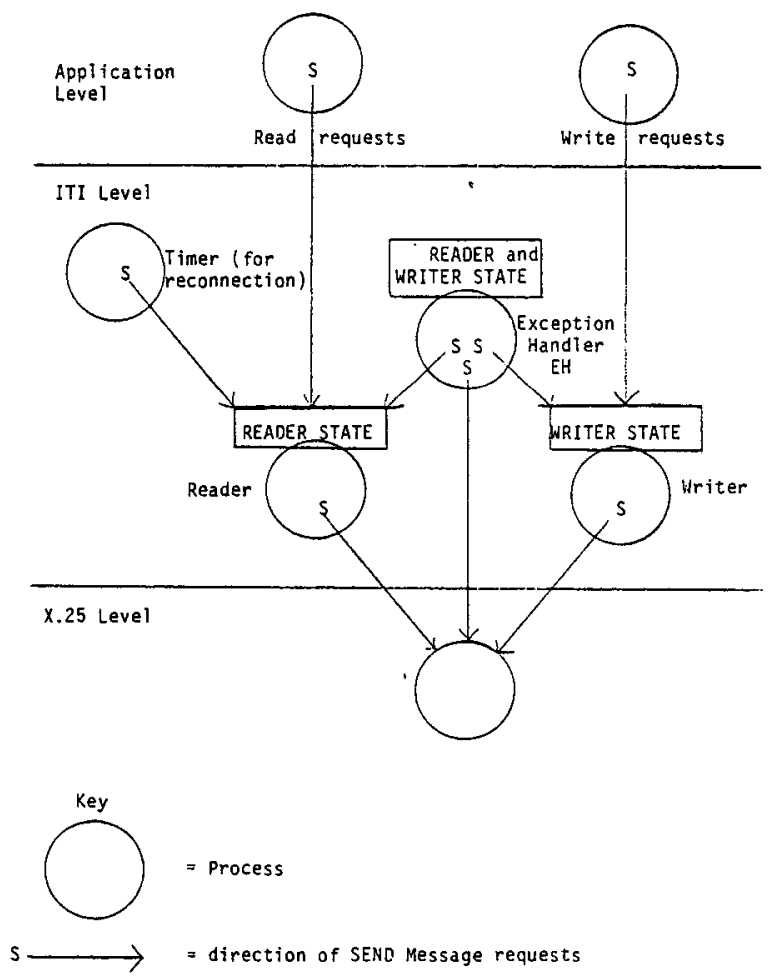

Figure 4. ITI as an $1 / 0$ filter

This structure reflects the major functionality of ITI, that of an I/O filter, better than the server model. Furthermore, it is structured more efficiently for the normal case (no interrupts or network failures) by actually reducing the process switches from 5 to 3 on each $\mathrm{read} / \mathrm{wr}$ ite request.

Exception processing occurs as follows. When an exception occurs, EH takes control by receiving a Reply from $\times .25$ which then "de-registers" $E H$. EH makes a copy of the relevent state information from the reader and writer workers, and then issues further read/write requests as appropriate, until the exceptional situation has been dealt with. EH then re-registers with $X .25$ and is ready to handle further exceptions. Exceptional events occurring across the X.25-ITI interface during this processing are queued up in $\times 25$ until there is a registered exception handler process ready to deal with them.

\subsection{Recovery after network failure}

There is one exception handler process, EH, for each $\times .25$ virtual circuit. If a network failure on a virtual circuit is recorded, the $\mathrm{EH}$ associated with the failed virtual circuit is notified immediately. It tears down the virtual circuit and suspends itself by executing a Send request to the process which would be invoked to handle a reconnection. If a timeout occurs before the remote user has reconnected, the timer destroys the suspended session, causing the user to be logged out.

If the user attempts to reconnect via a new $\times .25$ virtual circuit, another EH process is created for this circuit. The reconnection sequence involves a check of the remote user's status - if it is suspended, the old $E H$ associated with the torn-down virtual circuit is unblocked with a Reply. The old EH resets its session to the new $\times .25$ virtual circuit, and wakes up the reader and the writer, completing reconnection before destroying the new EH. The reader and writer then continue exactly as if the underlying network had not failed, replying with new data to the suspended application.

\section{Conclusions}

Two mechanisms for handling exceptions in multi-process protocol implementations have been described. The first mechanism reflects the use of combining the role of a server with a finite state machine. This structure is particularly efficient for the lower level protocols such as X.25, where it is difficult or impossible to define a normal flow of control.

For higher level protocols where exceptional situations occur less frequentl, it is more efficient to structure according to the normal flow of control. The server model is not so efficient in these situations instead, the protocol is restructured as an i/o filter with a special exception handler process. This structure combines effects of both synchronous and asynchronous exceptions; it can be generalised as a tool for other asynchronous problems, and it does not add overhead to the normal case. In fact it actually reduces overhead in the normal case through avoiding unnecessary context saving of global state information, and reducing the number of process switches on each read/write request.

These mechanisms can be generalised to programs other then protocol implementations, and to systems other than Verex. 


\section{Acknowledgements}

I am very grateful to David Cheriton for suggesting these studies of exception mechanisms, and for his continued interest and support of this work. I am also indebted to Steve Deering for the discussions that gave rise to many parts of this paper, and for his helpful comments throughout the production of this paper.

\section{References}

[1] Bochmann, G.V. Finite state description of communication protocols

Computer Networks 2, 4/5 (October 1978), $361-372$.

[2] CCITT Recommendation X.25 Interface between DTE and DCE for terminals operating in the packet mode on public data networks March 1976.

[3] Cheriton, D.R., Malcolm, M.A., Melen, L.S., and Sager, G.R. Thoth, a portable real-timeoperating system Communications of the ACM 22, 2 (February 1979), 105-115.

[4] Cheriton, D.R. Distributed I/O using an object-based protocol UBC Computer Science Technical Report 81-1, University of British Columbia, January 1981.

[5] Cheriton, D.R. The Thoth System: Multi-Process structuring and Portability

Elsevier North-Holland, New York, 1982.

[6] The Computer Communications Group; TransCanada Telephone System. Datapac Interactive Terminal Interface (ITI) Specification

Trans Canada Telephone System, Ottawa, Ontario, October 1978.

[7] Deering, S.E. Multi-process structuring of $X .25$ software UBC Computer Science Technical Report 82-11, University of British Columbia, October 1982.

[8] Lockhart, T.W. The design of a verifiable operating system kernel UBC Computer Science Technical Report 79-15, University of British Columbia, November 1979.

[9] Scotton, G.R. An experiment in High level protocol design University of British Columbia, Department of Computer Science, MSc. Thesis, December 1981. 